\title{
Some Hermite-Hadamard and Opial dynamic inequalities on time scales
}

\author{
Pshtiwan Othman Mohammed ${ }^{1 *} \mathbb{B}$, Cheon Seoung Ryoo ${ }^{2 *}$, Artion Kashuri ${ }^{3} \mathbb{D}$, Y.S. Hamed $^{4}$ (D) and \\ Khadijah M. Abualnaja ${ }^{4}$
}

${ }^{*}$ Correspondence:

pshtiwansangawi@gmail.com; ryoocs@hnu.kr

'Department of Mathematics, College of Education, University of Sulaimani, Sulaimani, Kurdistan Region, Iraq

${ }^{2}$ Department of Mathematics, Hannam University, Daejeon 34430, Korea

Full list of author information is available at the end of the article

\begin{abstract}
In this article, we are interested in some well-known dynamic inequalities on time scales. For this reason, we will prove some new Hermite-Hadamard $(\mathrm{H}-\mathrm{H})$ and Opial dynamic inequalities on time scales. The main results here will be derived via the dynamic integration by parts and chain rule formulas on time scales. In addition, we will extend and unify the inequalities for the convex functions.
\end{abstract}

MSC: 26D07; 26D10; 26D15; 26A33

Keywords: Time scales; $\mathrm{H}-\mathrm{H}$ inequality; Steffensen inequalities; Opial inequality; Hölder's inequality

\section{Introduction}

In 1893 , the $\mathrm{H}-\mathrm{H}$ inequality was established for a convex function $\wp$ on a given interval $\left[d_{1}, d_{2}\right]$ in $[1]$ :

$$
\wp\left(\frac{d_{1}+d_{2}}{2}\right) \leq \frac{1}{d_{2}-d_{1}} \int_{d_{1}}^{d_{2}} \wp(x) \mathrm{d} x \leq \frac{\wp\left(d_{1}\right)+\wp\left(d_{2}\right)}{2} .
$$

The study of the $\mathrm{H}-\mathrm{H}$ inequality have been attracted the attention of many scholars. In recent years, many refinements, generalizations, and extensions have been made to the inequality (1.1); we advise the interested reader to visit the published papers [2-8] and the references cited therein.

After the H-H inequality and in 1960, Opial [9] established another important integral inequality, called in the literature Opial's integral inequality, which is as follows:

$$
\int_{0}^{\mu}\left|\wp(\mathbf{s}) \wp^{\prime}(\mathbf{s})\right| \mathrm{d} \mathbf{s} \leq \frac{\mu}{4} \int_{0}^{\mu}\left(\wp^{\prime}(\mathbf{s})\right)^{2} \mathrm{~d} \mathbf{s},
$$

where $\wp(\mathbf{s}) \in C^{1}[0, \mu]$ with $\wp(0)=\wp(\mu)=0$ and $\wp(\mathbf{s})>0$ for $\mathbf{s} \in(0, \mu)$. A best possibility here is $\frac{\mu}{4}$. Inequality (1.2) with their extensions play a great role in analysis and its applications. The interest in inequality (1.2) comes from their mathematical structure. Many results concerning the generalizations and extensions of this inequality have been established; see [10-17].

(c) The Author(s) 2021. This article is licensed under a Creative Commons Attribution 4.0 International License, which permits use, sharing, adaptation, distribution and reproduction in any medium or format, as long as you give appropriate credit to the original author(s) and the source, provide a link to the Creative Commons licence, and indicate if changes were made. The images or other third party material in this article are included in the article's Creative Commons licence, unless indicated otherwise in a credit line to the material. If material is not included in the article's Creative Commons licence and your intended use is not permitted by statutory regulation or exceeds the permitted use, you will need to obtain permission directly from the copyright holder. To view a copy of this licence, visit http://creativecommons.org/licenses/by/4.0/. 
The inequalities (1.1) and (1.2) have been proved not only for the ordinary order but also for various fractional models, for example, the Riemann-Liouville model, the AtanganaBaleanu model, the tempered fractional model, the Caputo-Fabrizo model, and the conformable model; see [18-22] and the references therein.

The use of dynamic system to study the continuous and discrete times is well studied, especially for the real-world modeling issues. It is better to check if structures can be given that encourage us in integrating all dynamic systems at the same time to derive a superior and perspective comprehension of the contrasts between continuous and discrete domains. In fact, constructing a correlation between discrete and continuous situations is the primary aim of dynamic equations on time scales. It is well known that the theory of time scales was originated by Hilger in his Ph.D. thesis [23]. After that, this setting was evolved by many researchers, for more details refer to [24, 25].

Over the recent couple of years, there has been growing interest in the study of dynamic inequalities on time scales and this has become an important field in applied and pure mathematics; see for details [25-30].

This article is devoted to establishing some dynamic $\mathrm{H}-\mathrm{H}$ and Opial inequalities on time scales. The obtained inequalities will extend some known integral inequalities, and extend and unify some continuous inequalities.

The article consists of five sections. Section 1 is for the introduction. In Sect. 2 we present basic concepts and preliminaries of time scale notations, and in Sect. 3 we discuss and derive some dynamic inequalities of $\mathrm{H}-\mathrm{H}$ on time scales. Opial dynamic inequalities will be discussed in Sect. 4. Section 5 concludes the article finally.

\section{Preliminaries}

This section deals with recalling time scale notation and basic lemmas on Steffensen inequalities on time scales. Let $\mathrm{R}$ be the set of real numbers, then a time scale $T_{0}$ is a nonempty and closed subset of $\mathrm{R}$. For $\iota \in \mathrm{T}_{0}$, the forward and backward jump operators $\sigma, \rho: \mathrm{T}_{0} \rightarrow \mathrm{R}$ are, respectively, defined by

$$
\sigma(\iota)=\inf \left\{n \in \mathrm{T}_{0}: n>\iota\right\} \quad \text { and } \rho(\iota)=\sup \left\{n \in \mathrm{T}_{0}: n<\iota\right\}
$$

We define the graininess function $\wp: \mathrm{T}_{0} \rightarrow[0, \infty)$ by $\wp(\iota)=\sigma(\iota)-\iota$. An element $\iota \in \mathrm{T}_{0}$ is said to be left-dense if $\rho(\iota)=\iota$ and left-scattered if $\rho(\iota)<\iota$, and right-dense if $\sigma(\iota)=\iota$ and right-scattered if $\sigma(\iota)>\iota$. The set $\mathrm{T}_{0}{ }^{k}$ is defined to be $\mathrm{T}_{0}$ if it has a left-scattered maximum $\wp_{2}$, then $\mathrm{T}_{0}{ }^{k}=\mathrm{T}_{0}-\left\{\wp_{2}\right\}$ otherwise $\mathrm{T}_{0}{ }^{k}=\mathrm{T}_{0}$. For further information on these notions we refer the reader to Refs. [24, 25].

Definition 2.1 ([25]) Assume that $\wp: \mathrm{T}_{0} \rightarrow \mathrm{R}$ is a real-valued function. Then we say $\wp$ is $\mathcal{R D}$-continuous on $\mathrm{R}$ if its left limit is finite at any left-dense point of $\mathrm{T}_{0}$ and it is continuous on every right-dense point of $\mathrm{T}_{0}$.

Definition 2.2 ([25]) Assume that $\wp: \mathrm{T}_{0} \rightarrow \mathrm{R}$ is a real-valued function. Then we say $\wp$ is $\mathcal{L D}$-continuous on $\mathrm{R}$ if its right limit is finite at any right-dense point of $\mathrm{T}_{0}$ and it is continuous on every left-dense point of $\mathrm{T}_{0}$.

Theorem 2.1 ([25]) Let $d_{1}, d_{2} \in \mathrm{T}_{0}$ with $d_{1}<d_{2}$. Let $\wp_{1}, \wp_{2}:\left[d_{1}, d_{2}\right]_{\mathrm{T}_{0}} \rightarrow \mathrm{R}$ be $\Delta$ integrable functions such that $\wp_{1}$ of one sign and decreasing and $0 \leq \wp_{2}(\mathbf{s}) \leq 1$ for each 
$\mathbf{s} \in\left[d_{1}, d_{2}\right]_{\mathrm{T}_{0}}$. Also, suppose that $\vartheta_{1}, \vartheta_{2} \in\left[d_{1}, d_{2}\right]_{\mathrm{T}_{0}}$ such that

$$
\begin{aligned}
& d_{2}-\vartheta_{1} \leq \int_{d_{1}}^{d_{2}} \wp_{2}(\mathbf{s}) \Delta \mathbf{s} \leq \vartheta_{2}-d_{1}, \quad \text { if } \wp_{1}(\mathbf{s})>0, \forall \mathbf{s} \in\left[d_{1}, d_{2}\right]_{\mathrm{T}_{0}}, \\
& \vartheta_{2}-d_{1} \leq \int_{d_{1}}^{d_{2}} \wp_{2}(\mathbf{s}) \Delta \mathbf{s} \leq d_{2}-\vartheta_{1}, \quad \text { if } \wp_{1}(\mathbf{s})<0, \forall \mathbf{s} \in\left[d_{1}, d_{2}\right]_{\mathrm{T}_{0}},
\end{aligned}
$$

then

$$
\int_{\vartheta_{1}}^{d_{2}} \wp_{1}(\mathbf{s}) \Delta \mathbf{s} \leq \int_{d_{1}}^{d_{2}} \wp_{1}(\mathbf{s}) \wp_{2}(\mathbf{s}) \Delta \mathbf{s} \leq \int_{d_{1}}^{\vartheta_{2}} \wp_{1}(\mathbf{s}) \Delta \mathbf{s} .
$$

Theorem 2.2 ([25]) Let $d_{1}, d_{2} \in \mathrm{T}_{0}$ with $d_{1}<d_{2}$. Let $\wp_{1}, \wp_{2}:\left[d_{1}, d_{2}\right]_{\mathrm{T}_{0}} \rightarrow \mathrm{R}$ be $\nabla$ integrable functions such that $\wp_{1}$ is of one sign and decreasing and $0 \leq \wp_{2}(\mathbf{s}) \leq 1$ for each $\mathbf{s} \in\left[d_{1}, d_{2}\right]_{\mathrm{T}_{0}}$. Also, suppose that $\vartheta_{1}, \vartheta_{2} \in\left[d_{1}, d_{2}\right]_{\mathrm{T}_{0}}$ such that

$$
\begin{aligned}
& d_{2}-\vartheta_{1} \leq \int_{d_{1}}^{d_{2}} \wp_{2}(\mathbf{s}) \nabla \mathbf{s} \leq \vartheta_{2}-d_{1}, \quad \text { if } \wp_{1}(\mathbf{s})>0, \forall \mathbf{s} \in\left[d_{1}, d_{2}\right]_{\mathrm{T}_{0}}, \\
& \vartheta_{2}-d_{1} \leq \int_{d_{1}}^{d_{2}} \wp_{2}(\mathbf{s}) \nabla \mathbf{s} \leq d_{2}-\vartheta_{1}, \quad \text { if } \wp_{1}(\mathbf{s})<0, \forall \mathbf{s} \in\left[d_{1}, d_{2}\right]_{\mathrm{T}_{0}},
\end{aligned}
$$

then

$$
\int_{\vartheta_{1}}^{d_{2}} \wp_{1}(\mathbf{s}) \nabla \mathbf{s} \leq \int_{d_{1}}^{d_{2}} \wp_{1}(\mathbf{s}) \wp_{2}(\mathbf{s}) \nabla \mathbf{s} \leq \int_{d_{1}}^{\vartheta_{2}} \wp_{1}(\mathbf{s}) \nabla \mathbf{s} .
$$

Theorem 2.3 ([25]) Let $d_{1}, d_{2} \in \mathrm{T}_{0}$ with $d_{1}<d_{2}$. Let $\wp_{1}, \wp_{2}:\left[d_{1}, d_{2}\right]_{\mathrm{T}_{0}} \rightarrow \mathrm{R}$ be $\Delta$ integrable functions such that $\wp_{1}$ of one sign and decreasing and $0 \leq \wp_{2}(\mathbf{s}) \leq 1$ for each $\mathbf{s} \in\left[d_{1}, d_{2}\right]_{\mathrm{T}_{0}}$. Suppose that

$$
\lambda:=\int_{d_{1}}^{d_{2}} \wp_{2}(\mathbf{s}) \Delta \mathbf{s} \quad \text { provided that } d_{2}-\lambda, d_{1}+\lambda \in \mathrm{T}_{0},
$$

then

$$
\int_{d_{2}-\lambda}^{d_{2}} \wp_{1}(\mathbf{s}) \Delta \mathbf{s} \leq \int_{d_{1}}^{d_{2}} \wp_{1}(\mathbf{s}) \wp_{2}(\mathbf{s}) \Delta \mathbf{s} \leq \int_{d_{1}}^{d_{1}+\lambda} \wp_{1}(\mathbf{s}) \Delta \mathbf{s} .
$$

Theorem 2.4 ([25]) Let $d_{1}, d_{2} \in \mathrm{T}_{0}$ with $d_{1}<d_{2}$. Let $\wp_{1}, \wp_{2}:\left[d_{1}, d_{2}\right]_{\mathrm{T}_{0}} \rightarrow \mathrm{R}$ be $\nabla$ integrable functions such that $\wp_{1}$ of one sign and decreasing and $0 \leq \wp_{2}(\mathbf{s}) \leq 1$ for each $\mathbf{s} \in\left[d_{1}, d_{2}\right]_{\mathrm{T}_{0}}$. Suppose that

$$
\lambda:=\int_{d_{1}}^{d_{2}} \wp_{2}(\mathbf{s}) \nabla \mathbf{s} \quad \text { such that } d_{2}-\lambda, d_{1}+\lambda \in \mathrm{T}_{0},
$$

then

$$
\int_{d_{2}-\lambda}^{d_{2}} \wp_{1}(\mathbf{s}) \nabla \mathbf{s} \leq \int_{d_{1}}^{d_{2}} \wp_{1}(\mathbf{s}) \wp_{2}(\mathbf{s}) \nabla \mathbf{s} \leq \int_{d_{1}}^{d_{1}+\lambda} \wp_{1}(\mathbf{s}) \nabla \mathbf{s} .
$$


Theorem 2.5 ( $\Delta$-integration by parts $[24,25])$ Let $\wp_{1}, \wp_{2}:\left[d_{1}, d_{2}\right]_{T_{0}} \rightarrow \mathrm{R}$ with $\wp_{1}, \wp_{2} \in$ $\mathbb{C}_{\mathcal{R D}}$ and $d_{1}, d_{2} \in \mathrm{T}_{0}$. Then the integration by parts in the sense of $\Delta$ is formulated as follows:

$$
\int_{d_{1}}^{d_{2}} \wp_{1}(\mathbf{s}) \wp_{2}^{\Delta}(\mathbf{s}) \Delta \mathbf{s}=\left.\wp_{1}(\mathbf{s}) \wp_{2}(\mathbf{s})\right|_{d_{1}} ^{d_{2}}-\int_{d_{1}}^{d_{2}} \wp_{1}^{\Delta}(\mathbf{s}) \wp_{2}^{\sigma}(\mathbf{s}) \Delta \mathbf{s}
$$

Theorem 2.6 ( $\nabla$-integration by parts $[17,24,25])$ Let $\wp_{1}, \wp_{2}:\left[d_{1}, d_{2}\right]_{\mathrm{T}_{0}} \rightarrow \mathrm{R}$ with $\wp_{1}, \wp_{2} \in$ $\mathbb{C}_{\mathcal{L D}}$ and $d_{1}, d_{2} \in \mathrm{T}_{0}$. Then the integration by parts in the sense of $\nabla$ is formulated as follows:

$$
\int_{d_{1}}^{d_{2}} \wp_{1}(\mathbf{s}) \wp_{2}^{\nabla}(\mathbf{s}) \nabla \mathbf{s}=\left.\wp_{1}(\mathbf{s}) \wp_{2}(\mathbf{s})\right|_{d_{1}} ^{d_{2}}-\int_{d_{1}}^{d_{2}} \wp_{1}^{\nabla}(\mathbf{s}) \wp_{2}^{\rho}(\mathbf{s}) \nabla \mathbf{s}
$$

Definition 2.3 ([31]) Let $d_{1}, d_{2} \in \mathrm{T}_{0}$ with $d_{1}<d_{2}$. A function $\wp: \mathrm{T}_{0} \rightarrow \mathbb{R}$ is said to be convex on $\mathrm{T}_{0}$, if

$$
\wp\left(\vartheta d_{1}+(1-\vartheta) d_{2}\right) \leq \vartheta \wp\left(d_{1}\right)+(1-\vartheta) \wp\left(d_{2}\right)
$$

holds for each $\vartheta \in \mathrm{T}_{0\left[d_{1}, d_{2}\right]} \subseteq[0,1]$.

\section{Dynamic $\mathbf{H}-\mathrm{H}$ inequalities}

Theorem 3.1 Let $\wp:\left[d_{1}, d_{2}\right]_{\mathrm{T}_{0}} \rightarrow \mathrm{R}$ be convex and monotonic and $d_{1}, d_{2} \in \mathrm{T}_{0}$ with $d_{1}<d_{2}$. Suppose that also $\vartheta_{1}, \vartheta_{2} \in\left[d_{1}, d_{2}\right]_{\mathrm{T}_{0}}$, then we have

$$
\wp\left(\frac{\vartheta_{1}+\vartheta_{2}}{2}\right) \leq \frac{1}{d_{2}-d_{1}} \int_{d_{1}}^{d_{2}} \wp^{\sigma}(\mathbf{s}) \Delta \mathbf{s} \leq \frac{2 \wp\left(d_{1}\right)-\wp\left(\vartheta_{1}\right)-\wp\left(\vartheta_{2}\right)+2 \wp\left(d_{2}\right)}{2}
$$

such that $\frac{d_{1}+d_{2}}{2}, \frac{\vartheta_{1}+\vartheta_{2}}{2} \in \mathrm{T}_{0}$ and

$$
\begin{aligned}
& \vartheta_{1}, \vartheta_{2} \geq \frac{d_{1}+3 d_{2}}{4}, \quad \text { if } \wp \text { is decreasing, } \\
& \vartheta_{1}, \vartheta_{2} \leq \frac{d_{1}+3 d_{2}}{4}, \quad \text { if } \wp \text { is increasing. }
\end{aligned}
$$

Proof Suppose that $\wp$ is decreasing and convex. It follows that $\wp^{\Delta} \leq 0$. Set $\Psi_{1}:=-\wp^{\Delta}$, then it is clear that $\Psi_{1}$ is decreasing and $\Psi_{1} \geq 0$. If we choose $\Psi_{2}(\mathbf{s}):=\frac{2\left(d_{2}-\mathbf{s}\right)}{d_{2}-d_{1}}$, we see that $0 \leq \Psi_{2}(\mathbf{s}) \leq 1$ for each $\mathbf{s} \in\left[\frac{d_{1}+d_{2}}{2}, d_{2}\right]$. Now, by making use of inequality (2.1) with $\wp_{2}(\mathbf{s})=$ $\Psi_{2}(\mathbf{s})=\frac{2\left(d_{2}-\mathbf{s}\right)}{d_{2}-d_{1}}$, we get

$$
d_{2}-\vartheta_{1} \leq \frac{d_{2}-d_{1}}{4} \leq \vartheta_{2}-\frac{d_{1}+d_{2}}{2}
$$

This implies that $\vartheta_{1}, \vartheta_{2} \geq \frac{d_{1}+3 d_{2}}{4}$. Thus, $\Psi_{1}$ and $\Psi_{2}$ satisfy the hypotheses in Theorem 2.1 and therefore

$$
\int_{\vartheta_{1}}^{d_{2}} \Psi_{1}(\mathbf{s}) \Delta \mathbf{s} \leq \int_{\frac{d_{1}+d_{2}}{2}}^{d_{2}} \Psi_{1}(\mathbf{s}) \Psi_{2}(\mathbf{s}) \Delta \mathbf{s} \leq \int_{\frac{d_{1}+d_{2}}{2}}^{\vartheta_{2}} \Psi_{1}(\mathbf{s}) \Delta \mathbf{s}
$$


By using $\Delta$-integration by parts (Theorem 2.5 ), we have

$$
\int_{\frac{d_{1}+d_{2}}{2}}^{d_{2}} \Psi_{1}(\mathbf{s}) \Psi_{2}(\mathbf{s}) \Delta \mathbf{s}=\wp\left(\frac{d_{1}+d_{2}}{2}\right)-\frac{2}{d_{2}-d_{1}} \int_{\frac{d_{1}+d_{2}}{2}}^{d_{2}} \wp^{\sigma}(\mathbf{s}) \Delta \mathbf{s} .
$$

Then, by making use of the above and the fact $\int_{x_{1}}^{x_{2}} \Psi_{1}(\mathbf{s}) \Delta \mathbf{s}=\wp\left(x_{1}\right)-\wp\left(x_{2}\right)$ in the inequality (3.2), we get

$$
\wp\left(\vartheta_{1}\right)-\wp\left(d_{2}\right) \leq \wp\left(\frac{d_{1}+d_{2}}{2}\right)-\frac{2}{d_{2}-d_{1}} \int_{\frac{d_{1}+d_{2}}{2}}^{d_{2}} \wp^{\sigma}(\mathbf{s}) \Delta \mathbf{s} \leq \wp\left(\frac{d_{1}+d_{2}}{2}\right)-\wp\left(\vartheta_{2}\right) .
$$

This simplifies to

$$
\wp\left(\vartheta_{2}\right) \leq \frac{2}{d_{2}-d_{1}} \int_{\frac{d_{1}+d_{2}}{2}}^{d_{2}} \wp^{\sigma}(\mathbf{s}) \Delta \mathbf{s} \leq \frac{\wp\left(d_{1}\right)+\wp\left(d_{2}\right)}{2}+\wp\left(d_{2}\right)-\wp\left(\vartheta_{1}\right)
$$

where we used the convexity of $\wp$ to get $\wp\left(\frac{d_{1}+d_{2}}{2}\right) \leq \frac{\wp\left(d_{1}\right)+\wp\left(d_{2}\right)}{2}$.

On the other hand, if we choose $\Psi_{3}(\mathbf{s}):=\frac{2\left(\mathbf{s}-d_{1}\right)}{d_{2}-d_{1}}$, we see that $0 \leq \Psi_{3}(\mathbf{s}) \leq 1$ for each $\mathbf{s} \in\left[d_{1}, \frac{d_{1}+d_{2}}{2}\right]$. Again, by making use of inequality (2.1) for the new $\Psi_{3}(\mathbf{s})$, we find

$$
\frac{d_{1}+d_{2}}{2}-\vartheta_{1} \leq \frac{d_{2}-d_{1}}{4} \leq \vartheta_{2}-d_{1}
$$

This implies that $\vartheta_{1}, \vartheta_{2} \geq \frac{3 d_{1}+d_{2}}{4}<\frac{d_{1}+3 d_{2}}{4}$. Thus, $\Psi_{1}$ and $\Psi_{3}$ satisfy the hypotheses in Theorem 2.1. Then, by using the same technique as used above, we can deduce

$$
\wp\left(\vartheta_{1}\right) \leq \frac{2}{d_{2}-d_{1}} \int_{d_{1}}^{\frac{d_{1}+d_{2}}{2}} \wp^{\sigma}(\mathbf{s}) \Delta \mathbf{s} \leq \wp\left(d_{1}\right)+\wp\left(\frac{d_{1}+d_{2}}{2}\right)-\wp\left(\vartheta_{2}\right) .
$$

By convexity of $\wp$, it follows that

$$
\wp\left(\vartheta_{1}\right) \leq \frac{2}{d_{2}-d_{1}} \int_{d_{1}}^{\frac{d_{1}+d_{2}}{2}} \wp^{\sigma}(\mathbf{s}) \Delta \mathbf{s} \leq \wp\left(d_{1}\right)+\frac{\wp\left(d_{1}\right)+\wp\left(d_{2}\right)}{2}-\wp\left(\vartheta_{2}\right) .
$$

Adding inequalities (3.3) and (3.4) together and simplifying the result we get

$$
\frac{\wp\left(\vartheta_{2}\right)+\wp\left(\vartheta_{1}\right)}{2} \leq \frac{1}{d_{2}-d_{1}} \int_{d_{1}}^{d_{2}} \wp^{\sigma}(\mathbf{s}) \Delta \mathbf{s} \leq \frac{2 \wp\left(d_{1}\right)+2 \wp\left(d_{2}\right)}{2}-\frac{\wp\left(\vartheta_{2}\right)+\wp\left(\vartheta_{1}\right)}{2}
$$

Again, by using the convexity of $\wp$ (we see $\left.\wp\left(\frac{\vartheta_{2}+\vartheta_{1}}{2}\right) \leq \frac{\wp\left(\vartheta_{2}\right)+\wp\left(\vartheta_{1}\right)}{2}\right)$ for the last inequality and rearranging the terms, we get the desired result.

Corollary 3.1 Theorem 3.1 with $\vartheta_{1}=\vartheta_{2}=\frac{d_{1}+3 d_{2}}{4}$ gives the new inequality

$$
\wp\left(\frac{d_{1}+3 d_{2}}{4}\right) \leq \frac{1}{d_{2}-d_{1}} \int_{d_{1}}^{d_{2}} \wp^{\sigma}(\mathbf{s}) \Delta \mathbf{s} \leq \frac{2 \wp\left(d_{1}\right)+2 \wp\left(d_{2}\right)}{2}-\wp\left(\frac{d_{1}+3 d_{2}}{4}\right) .
$$


Theorem 3.2 Let $\wp:\left[d_{1}, d_{2}\right]_{\mathrm{T}_{0}} \rightarrow \mathrm{R}$ be convex and monotonic and $d_{1}, d_{2} \in \mathrm{T}_{0}$ with $d_{1}<d_{2}$, then we have

$$
\wp\left(\frac{d_{1}+d_{2}}{2}\right) \leq \frac{1}{d_{2}-d_{1}} \int_{d_{1}}^{d_{2}} \wp^{\sigma}(\mathbf{s}) \Delta \mathbf{s} \leq \frac{2 \wp\left(d_{1}\right)+2 \wp\left(d_{2}\right)}{2}-\wp\left(\frac{d_{1}+d_{2}}{2}\right),
$$

such that $\frac{d_{1}+d_{2}}{2} \in \mathrm{T}_{0}$.

Proof Suppose that $\wp$ is decreasing and convex. It follows that $\wp^{\Delta} \leq 0$. Let $\Psi_{1}:=-\wp^{\Delta}$, then we see that $\Psi_{1}$ is decreasing and $\Psi_{1} \geq 0$. If we choose $\Psi_{2}(\mathbf{s}):=\frac{d_{2}-\mathbf{s}}{d_{2}-d_{1}}$, we see that $0 \leq \Psi_{2}(\mathbf{s}) \leq 1$ for each $\mathbf{s} \in\left[d_{1}, d_{2}\right]$. Moreover,

$$
\lambda:=\int_{d_{1}}^{d_{2}} \Psi_{2}(\mathbf{s}) \Delta \mathbf{s}=\frac{d_{2}-d_{1}}{2} .
$$

It follows that $d_{1}+\lambda=d_{2}-\lambda=\frac{d_{1}+d_{2}}{2} \in \mathrm{T}_{0}$. Thus, $\Psi_{1}$ and $\Psi_{2}$ satisfy the hypotheses in Theorem 2.3 and therefore inequality (2.5) holds true for $\Psi_{1}=-\wp^{\Delta}$ and $\Psi_{2}(\mathbf{s})=\frac{d_{2}-\mathbf{s}}{d_{2}-d_{1}}$.

$$
\int_{\frac{d_{1}+d_{2}}{2}}^{d_{2}} \Psi_{1}(\mathbf{s}) \Delta \mathbf{s} \leq \int_{d_{1}}^{d_{2}} \Psi_{1}(\mathbf{s}) \Psi_{2}(\mathbf{s}) \Delta \mathbf{s} \leq \int_{d_{1}}^{\frac{d_{1}+d_{2}}{2}} \Psi_{1}(\mathbf{s}) \Delta \mathbf{s} .
$$

By making use of integration by parts and the fact $\int_{x_{1}}^{x_{2}} \Psi_{1}(\mathbf{s}) \Delta \mathbf{s}=\wp\left(x_{1}\right)-\wp\left(x_{2}\right)$, we can deduce

$$
\wp\left(\frac{d_{1}+d_{2}}{2}\right)-\wp\left(d_{2}\right) \leq \wp\left(d_{1}\right)-\frac{1}{d_{2}-d_{1}} \int_{d_{1}}^{d_{2}} \wp^{\sigma}(\mathbf{s}) \Delta \mathbf{s} \leq \wp\left(d_{1}\right)-\wp\left(\frac{d_{1}+d_{2}}{2}\right),
$$

which rearranges to the desired result.

The above results can be obtained for the $\nabla$ case by using Theorems 2.2 and 2.4, respectively.

Theorem 3.3 Let $\wp:\left[d_{1}, d_{2}\right]_{\mathrm{T}_{0}} \rightarrow \mathrm{R}$ be convex and monotonic and $d_{1}, d_{2} \in \mathrm{T}_{0}$ with $d_{1}<d_{2}$. Suppose that also $\vartheta_{1}, \vartheta_{2} \in\left[d_{1}, d_{2}\right]_{T_{0}}$, then we have

$$
\wp\left(\frac{\vartheta_{1}+\vartheta_{2}}{2}\right) \leq \frac{1}{d_{2}-d_{1}} \int_{d_{1}}^{d_{2}} \wp^{\rho}(\mathbf{s}) \nabla \mathbf{s} \leq \frac{2 \wp\left(d_{1}\right)-\wp\left(\vartheta_{1}\right)-\wp\left(\vartheta_{2}\right)+2 \wp\left(d_{2}\right)}{2},
$$

such that $\frac{d_{1}+d_{2}}{2}, \frac{\vartheta_{1}+\vartheta_{2}}{2} \in \mathrm{T}_{0}$ and

$$
\begin{aligned}
& \vartheta_{1}, \vartheta_{2} \geq \frac{d_{1}+3 d_{2}}{4}, \quad \text { if } \wp \text { is decreasing, } \\
& \vartheta_{1}, \vartheta_{2} \leq \frac{d_{1}+3 d_{2}}{4}, \quad \text { if } \wp \text { is increasing. }
\end{aligned}
$$

Corollary 3.2 Theorem 3.3 with $\vartheta_{1}=\vartheta_{2}=\frac{d_{1}+3 d_{2}}{4}$ gives the new inequality

$$
\wp\left(\frac{d_{1}+3 d_{2}}{4}\right) \leq \frac{1}{d_{2}-d_{1}} \int_{d_{1}}^{d_{2}} \wp^{\rho}(\mathbf{s}) \nabla \mathbf{s} \leq \frac{2 \wp\left(d_{1}\right)+2 \wp\left(d_{2}\right)}{2}-\wp\left(\frac{d_{1}+3 d_{2}}{4}\right) .
$$


Theorem 3.4 Let $\wp:\left[d_{1}, d_{2}\right]_{\mathrm{T}_{0}} \rightarrow \mathrm{R}$ be convex and monotonic and $d_{1}, d_{2} \in \mathrm{T}_{0}$ with $d_{1}<d_{2}$, then we have

$$
\wp\left(\frac{d_{1}+d_{2}}{2}\right) \leq \frac{1}{d_{2}-d_{1}} \int_{d_{1}}^{d_{2}} \wp^{\rho}(\mathbf{s}) \nabla \mathbf{s} \leq \frac{2 \wp\left(d_{1}\right)+2 \wp\left(d_{2}\right)}{2}-\wp\left(\frac{d_{1}+d_{2}}{2}\right)
$$

such that $\frac{d_{1}+d_{2}}{2} \in \mathrm{T}_{0}$.

\section{Dynamic Opial inequalities}

Theorem 4.1 Let $0, \mu \in \mathrm{T}_{0}$. For a delta differentiable and increasing function $\wp$ : $[0, \mu]_{\mathrm{T}_{0}} \rightarrow \mathrm{R}$ with $\wp(0)=0$, then

$$
|\wp(\mu)|\left(|\wp(\mu)|-\left|\wp\left(\frac{\mu}{2}\right)\right|\right) \leq \int_{0}^{\mu}|\wp(\mathbf{s})|\left|\wp^{\Delta}(\mathbf{s})\right| \Delta \mathbf{s} \leq|\wp(\mu)|\left|\wp\left(\frac{\mu}{2}\right)\right|,
$$

with equality when $\wp(\mathbf{s})=c \mathbf{s}$, for some $c \in \mathrm{R}$.

Proof Let $\Psi_{1}(\mathbf{s}):=\left|\wp^{\Delta}(\mathbf{s})\right|$, then $\Psi_{1}(\mathbf{s}) \geq 0$ for all $\mathbf{s} \in[0, \mu]$. Taking $\Psi_{2}(\mathbf{s}):=\frac{|\wp(\mathbf{s})|}{|\wp(\mu)|}$ and since $\wp(\mathbf{s})$ is an increasing function on $[0, \mu]$, we see that $0 \leq \Psi_{2}(\mathbf{s}) \leq 1$ for each $\mathbf{s} \in[0, \mu]$. Thus, $\Psi_{1}$ and $\Psi_{2}$ satisfy the hypotheses in Theorem 2.3 . Hence

$$
\int_{\frac{\mu}{2}}^{\mu} \Psi_{1}(\mathbf{s}) \Delta \mathbf{s} \leq \int_{0}^{\mu} \Psi_{1}(\mathbf{s}) \Psi_{2}(\mathbf{s}) \Delta \mathbf{s} \leq \int_{0}^{\frac{\mu}{2}} \Psi_{1}(\mathbf{s}) \Delta \mathbf{s} .
$$

So,

$$
\int_{\frac{\mu}{2}}^{\mu}\left|\wp^{\Delta}(\mathbf{s})\right| \Delta \mathbf{s} \leq \frac{1}{|\wp(\mu)|} \int_{0}^{\mu}|\wp(\mathbf{s})|\left|\wp^{\Delta}(\mathbf{s})\right| \Delta \mathbf{s} \leq \int_{0}^{\frac{\mu}{2}}\left|\wp^{\Delta}(\mathbf{s})\right| \Delta \mathbf{s} .
$$

By making use of integration by parts and the fact

$$
\int_{a_{1}}^{a_{2}} \Psi_{1}(\mathbf{s}) \Delta \mathbf{s}=\left|\wp\left(a_{2}\right)\right|-\left|\wp\left(a_{1}\right)\right|
$$

we get

$$
|\wp(\mu)|-\left|\wp\left(\frac{\mu}{2}\right)\right| \leq \frac{1}{|\wp(\mu)|} \int_{0}^{\mu}|\wp(\mathbf{s})|\left|\wp^{\Delta}(\mathbf{s})\right| \Delta \mathbf{s} \leq\left|\wp\left(\frac{\mu}{2}\right)\right|-|\wp(0)| .
$$

Multiplying inequality (4.2) on both sides by the factor $|\wp(\mu)|>0$ and from the condition $\wp(0)=0$ we obtain the desired result (4.1). Now, let $\wp(\mathbf{s})=c$ s for some $c \in R$. Then $\wp^{\Delta}(\mathbf{s})=$ $c$ and it is easy to check that equality holds in (4.1). The proof is complete.

Theorem 4.2 Let $d_{1}, d_{2} \in \mathrm{T}_{0}$ and $d_{1}<d_{2}$. Assume that $\wp, \wp^{\sigma}, \wp^{\Delta} \in \mathbb{C}_{\mathcal{R D}}\left(\left[d_{1}, d_{2}\right]_{\mathrm{T}_{0}}, \mathrm{R}\right)$ and $p>1$. Then

$$
\begin{aligned}
\left(\int_{d_{1}}^{d_{2}}\left|\wp(\mathbf{s})+\wp^{\sigma}(\mathbf{s})\right|^{p}\left|\wp^{\Delta}(\mathbf{s})\right| \Delta \mathbf{s}\right)^{\frac{1}{p}} \leq & \left(\int_{d_{1}}^{d_{2}}|\wp(\mathbf{s})|^{p}\left|\wp^{\Delta}(\mathbf{s})\right| \Delta \mathbf{s}\right)^{\frac{1}{p}} \\
& +\left(\int_{d_{1}}^{d_{2}}\left|\wp^{\sigma}(\mathbf{s})\right|^{p}\left|\wp^{\Delta}(\mathbf{s})\right| \Delta \mathbf{s}\right)^{\frac{1}{p}} .
\end{aligned}
$$


Proof Note

$$
\begin{aligned}
\int_{d_{1}}^{d_{2}}\left|\wp(\mathbf{s})+\wp^{\sigma}(\mathbf{s})\right|^{p}\left|\wp^{\Delta}(\mathbf{s})\right| \Delta \mathbf{s} \\
=\int_{d_{1}}^{d_{2}}\left|\wp(\mathbf{s})+\wp^{\sigma}(\mathbf{s})\right|^{p-1}\left|\wp(\mathbf{s})+\wp^{\sigma}(\mathbf{s})\right|\left|\wp^{\Delta}(\mathbf{s})\right| \Delta \mathbf{s} \\
\leq \int_{d_{1}}^{d_{2}}\left|\wp(\mathbf{s})+\wp^{\sigma}(\mathbf{s})\right|^{p-1}|\wp(\mathbf{s})|\left|\wp^{\Delta}(\mathbf{s})\right| \Delta \mathbf{s} \\
\quad+\int_{d_{1}}^{d_{2}}\left|\wp(\mathbf{s})+\wp^{\sigma}(\mathbf{s})\right|^{p-1}\left|\wp^{\sigma}(\mathbf{s})\right|\left|\wp^{\Delta}(\mathbf{s})\right| \Delta \mathbf{s} .
\end{aligned}
$$

Applying the Hölder inequality, we get

$$
\begin{aligned}
\int_{d_{1}}^{d_{2}}\left|\wp(\mathbf{s})+\wp^{\sigma}(\mathbf{s})\right|^{p}\left|\wp^{\Delta}(\mathbf{s})\right| \Delta \mathbf{s} \\
\leq\left(\int_{d_{1}}^{d_{2}}\left(\left|\wp(\mathbf{s})+\wp^{\sigma}(\mathbf{s})\right|^{p-1}\right)^{q}\left|\wp^{\Delta}(\mathbf{s})\right| \Delta \mathbf{s}\right)^{\frac{1}{q}}\left(\int_{d_{1}}^{d_{2}}|\wp(\mathbf{s})|^{p}\left|\wp^{\Delta}(\mathbf{s})\right| \Delta \mathbf{s}\right)^{\frac{1}{p}} \\
+\left(\int_{d_{1}}^{d_{2}}\left(\left|\wp(\mathbf{s})+\wp^{\sigma}(\mathbf{s})\right|^{p-1}\right)^{q}\left|\wp^{\Delta}(\mathbf{s})\right| \Delta \mathbf{s}\right)^{\frac{1}{q}}\left(\int_{d_{1}}^{d_{2}}\left|\wp^{\sigma}(\mathbf{s})\right|^{p}\left|\wp^{\Delta}(\mathbf{s})\right| \Delta \mathbf{s}\right)^{\frac{1}{p}} \\
=\left(\int_{d_{1}}^{d_{2}}\left|\wp(\mathbf{s})+\wp^{\sigma}(\mathbf{s})\right|^{p}\left|\wp^{\Delta}(\mathbf{s})\right| \Delta \mathbf{s}\right)^{\frac{1}{q}} \\
\quad \times\left[\left(\int_{d_{1}}^{d_{2}}|\wp(\mathbf{s})|^{p}\left|\wp^{\Delta}(\mathbf{s})\right| \Delta \mathbf{s}\right)^{\frac{1}{p}}+\left(\int_{d_{1}}^{d_{2}}\left|\wp^{\sigma}(\mathbf{s})\right|^{p}\left|\wp^{\Delta}(\mathbf{s})\right| \Delta \mathbf{s}\right)^{\frac{1}{p}}\right] .
\end{aligned}
$$

Therefore

$$
\begin{aligned}
& \left(\int_{d_{1}}^{d_{2}}\left|\wp(\mathbf{s})+\wp^{\sigma}(\mathbf{s})\right|^{p}\left|\wp^{\Delta}(\mathbf{s})\right| \Delta \mathbf{s}\right)^{\frac{1}{p}} \\
& \quad=\left(\int_{d_{1}}^{d_{2}}\left|\wp(\mathbf{s})+\wp^{\sigma}(\mathbf{s})\right|^{p}\left|\wp^{\Delta}(\mathbf{s})\right| \Delta \mathbf{s}\right)^{1-\frac{1}{q}} \\
& \leq\left[\left(\int_{d_{1}}^{d_{2}}|\wp(\mathbf{s})|^{p}\left|\wp^{\Delta}(\mathbf{s})\right| \Delta \mathbf{s}\right)^{\frac{1}{p}}+\left(\int_{d_{1}}^{d_{2}}\left|\wp^{\sigma}(\mathbf{s})\right|^{p}\left|\wp^{\Delta}(\mathbf{s})\right| \Delta \mathbf{s}\right)^{\frac{1}{p}}\right],
\end{aligned}
$$

which is the desired inequality (4.3). The proof is completed.

By making use of Theorem 4.1 and the well-known inequality

$$
\left|d_{1}+d_{2}\right|^{p} \leq 2^{p-1}\left(\left|d_{1}\right|^{p}+\left|d_{2}\right|^{p}\right), \quad p \geq 1,
$$

we can obtain the following result. 
Corollary 4.1 Let $0, \mu \in \mathrm{T}_{0}$. Assume that $\wp, \wp^{\sigma}, \wp^{\Delta} \in \mathbb{C}_{\mathcal{R D}}\left([0, \mu]_{\mathrm{T}_{0}}\right.$, R). For a delta differentiable and increasing function $\wp:[0, \mu]_{\mathrm{T}_{0}} \rightarrow \mathrm{R}$ with $\wp(0)=\wp^{\sigma}(0)=0$ and $p \geq 1$, then

$$
\int_{0}^{\mu}\left|\wp(\mathbf{s})+\wp^{\sigma}(\mathbf{s})\right|^{p}\left|\wp^{\Delta}(\mathbf{s})\right| \Delta \mathbf{s} \leq 2^{p-1}\left|\wp\left(\frac{\mu}{2}\right)\right|\left(|\wp(\mu)|^{p}+\left|\wp^{\sigma}(\mu)\right|^{p}\right) .
$$

By making use of Theorems 4.1 and 4.2, we can deduce the following inequality.

Corollary 4.2 Let $0, \mu \in \mathrm{T}_{0}$. Assume that $\wp, \wp^{\sigma}, \wp^{\Delta} \in \mathbb{C}_{\mathcal{R D}}\left([0, \mu]_{\mathrm{T}_{0}}, \mathrm{R}\right)$. For a delta differentiable and increasing function $\wp:[0, \mu]_{\mathrm{T}_{0}} \rightarrow \mathrm{R}$ with $\wp(0)=\wp^{\sigma}(0)=0$ and $p>1$, then

$$
\left(\int_{0}^{\mu}\left|\wp(\mathbf{s})+\wp^{\sigma}(\mathbf{s})\right|^{p}\left|\wp^{\Delta}(\mathbf{s})\right| \Delta \mathbf{s}\right)^{\frac{1}{p}} \leq\left|\wp\left(\frac{\mu}{2}\right)\right|^{\frac{1}{p}}\left(|\wp(\mu)|+\left|\wp^{\sigma}(\mu)\right|\right) .
$$

The above results can be obtained for the $\nabla$ case by using Theorems 2.2 and 2.4, respectively.

Theorem 4.3 Let $0, \mu \in \mathrm{T}_{0}$. For a nabla differentiable and increasing function $\wp$ : $[0, \mu]_{\mathrm{T}_{0}} \rightarrow \mathrm{R}$ with $\wp(0)=0$, then

$$
|\wp(\mu)|\left(|\wp(\mu)|-\left|\wp\left(\frac{\mu}{2}\right)\right|\right) \leq \int_{0}^{\mu}|\wp(\mathbf{s})|\left|\wp^{\nabla}(\mathbf{s})\right| \nabla \mathbf{s} \leq|\wp(\mu)| \wp\left(\frac{\mu}{2}\right) \mid,
$$

with equality when $\wp(\mathbf{s})=c \mathbf{s}$, for some $c \in \mathrm{R}$.

Theorem 4.4 Let $d_{1}, d_{2} \in \mathrm{T}_{0}$ and $d_{1}<d_{2}$. Assume that $\wp, \wp^{\rho}, \wp^{\nabla} \in \mathbb{C}_{\mathcal{L D}}\left(\left[d_{1}, d_{2}\right]_{\mathrm{T}_{0}}\right.$, R) and $p>1$. Then

$$
\begin{aligned}
\left(\int_{d_{1}}^{d_{2}}\left|\wp(\mathbf{s})+\wp^{\rho}(\mathbf{s})\right|^{p}\left|\wp^{\nabla}(\mathbf{s})\right| \nabla \mathbf{s}\right)^{\frac{1}{p}} \leq & \left(\int_{d_{1}}^{d_{2}}|\wp(\mathbf{s})|^{p}\left|\wp^{\nabla}(\mathbf{s})\right| \nabla \mathbf{s}\right)^{\frac{1}{p}} \\
& +\left(\int_{d_{1}}^{d_{2}}\left|\wp^{\rho}(\mathbf{s})\right|^{p}\left|\wp^{\nabla}(\mathbf{s})\right| \nabla \mathbf{s}\right)^{\frac{1}{p}} .
\end{aligned}
$$

By making use of Theorems 4.3 and 4.4, we can deduce the following inequalities, respectively.

Corollary 4.3 Let $0, \mu \in \mathrm{T}_{0}$. Assume that $\wp, \wp^{\rho}, \wp^{\nabla} \in \mathbb{C}_{\mathcal{L D}}\left([0, \mu]_{\mathrm{T}_{0}}, \mathrm{R}\right)$. For a nabla differentiable and increasing function $\wp:[0, \mu]_{\mathrm{T}_{0}} \rightarrow \mathrm{R}$ with $\wp(0)=\wp^{\rho}(0)=0$ and $p>1$, then

$$
\left(\int_{0}^{\mu}\left|\wp(\mathbf{s})+\wp^{\rho}(\mathbf{s})\right|^{p}\left|\wp^{\nabla}(\mathbf{s})\right| \nabla \mathbf{s}\right)^{\frac{1}{p}} \leq\left|\wp\left(\frac{\mu}{2}\right)\right|^{\frac{1}{p}}\left(|\wp(\mu)|+\left|\wp^{\rho}(\mu)\right|\right) .
$$

Corollary 4.4 Let $0, \mu \in \mathrm{T}_{0}$. Assume that $\wp, \wp^{\rho}, \wp^{\nabla} \in \mathbb{C}_{\mathcal{L D}}\left([0, \mu]_{\mathrm{T}_{0}}, \mathrm{R}\right)$. For a nabla differentiable and increasing function $\wp:[0, \mu]_{\mathrm{T}_{0}} \rightarrow \mathrm{R}$ with $\wp(0)=\wp^{\rho}(0)=0$ and $p \geq 1$, then

$$
\int_{0}^{\mu}\left|\wp(\mathbf{s})+\wp^{\rho}(\mathbf{s})\right|^{p}\left|\wp^{\nabla}(\mathbf{s})\right| \nabla \mathbf{s} \leq 2^{p-1}\left|\wp\left(\frac{\mu}{2}\right)\right|\left(|\wp(\mu)|^{p}+\left|\wp^{\rho}(\mu)\right|^{p}\right) .
$$




\section{Conclusion}

In this article, by making use of the well-known dynamic inequalities, a dynamic version of integration by parts and chain rule formulas, we obtained some useful dynamic $\mathrm{H}-\mathrm{H}$ and Opial inequalities on time scales. The derived inequalities generalize some well-known dynamic inequalities in the literature. For this purpose, the reader can see corollaries and remarks after each theorem of the main results.

\section{Acknowledgements}

This Research was supported by Taif University Researchers Supporting Project Number (TURSP-2020/217), Taif University, Taif, Saudi Arabia, and the National Research Foundation of Korea(NRF) grant funded by the Korea government(MEST) (No. 2017R1A2B4006092)

Funding

Not applicable.

Availability of data and materials

No data were used to support this study.

\section{Competing interests}

The authors declare that they have no competing interests.

Authors' contributions

All authors contributed equally and significantly in writing this article. All authors read and approved the final manuscript.

\section{Author details}

${ }^{1}$ Department of Mathematics, College of Education, University of Sulaimani, Sulaimani, Kurdistan Region, Iraq. ${ }^{2}$ Department of Mathematics, Hannam University, Daejeon 34430, Korea. ${ }^{3}$ Department of Mathematics, Faculty of Technical Science, University Ismail Qemali, Vlora, Albania. ${ }^{4}$ Department of Mathematics and Statistics, College of Science, Taif University, P.O. Box 11099, Taif 21944, Saudi Arabia.

\section{Publisher's Note}

Springer Nature remains neutral with regard to jurisdictional claims in published maps and institutional affiliations.

Received: 17 February 2021 Accepted: 27 April 2021 Published online: 05 May 2021

\section{References}

1. Hadamard, J.: Étude sur les propriétés des fonctions entières en particulier d'une fonction considérée par Riemann. J. Math. Pures Appl. 58, 171-215 (1893)

2. Sarikaya, M.Z., Set, E., Yaldiz, H., Başak, N.: Hermite-Hadamard's inequalities for fractional integrals and related fractional inequalities. Math. Comput. Model. 57, 2403-2407 (2013)

3. Srivastava, H.M., Zhang, Z.-H., Wu, Y.-D.: Some further refinements and extensions of the Hermite-Hadamard and Jensen inequalities in several variables. Math. Comput. Model. 54, 2709-2717 (2011)

4. Han, J., Mohammed, P.O., Zeng, H.: Generalized fractional integral inequalities of Hermite-Hadamard-type for a convex function. Open Math. 18, 794-806 (2020)

5. Kashuri, A., Meftah, B., Mohammed, P.O.: Some weighted Simpson type inequalities for differentiable s-convex functions and their applications. J. Frac. Calc. Nonlinear Sys. 1, 75-94 (2021)

6. Mohammed, P.O., Abdeljawad, T., Zeng, S., Kashuri, A.: Fractional Hermite-Hadamard integral inequalities for a new class of convex functions. Symmetry 12, 1485 (2020)

7. Mohammed, P.O., Aydi, H., Kashuri, A., Hamed, Y.S., Abualnaja, K.M.: Midpoint inequalities in fractional calculus defined using positive weighted symmetry function kernels. Symmetry 13, 550 (2021)

8. Khan, M.B., Mohammed, P.O., Noor, B., Hamed, Y.S.: New Hermite-Hadamard inequalities in fuzzy-interval fractional calculus and related inequalities. Symmetry 13,673 (2021)

9. Opial, Z:: Sur une inégalité. Ann. Pol. Math. 8, 29-32 (1960)

10. Zhao, C.-J., Cheung, W.-S.: On some Opial-type inequalities. J. Inequal. Appl. 2011, 7 (2011)

11. Samraiz, M., lqbal, S., Pečaric, J.: Generalized integral inequalities for fractional calculus. Cogent Math. Stat. 5, 1426205 (2018)

12. Başcı, Y., Baleanu, D.: New aspects of Opial-type integral inequalities. Adv. Differ. Equ. 2018, 452 (2018)

13. Sarikaya, M.Z., Bilisik, C.C., Mohammed, P.O.: Some generalizations of Opial type inequalities. Appl. Math. Inf. Sci. 14, 809-816 (2020)

14. Srivastava, H.M.: Some weighted Opial-type inequalities on time scale. Taiwan. J. Math. 14, 107-122 (2010)

15. KH, F.M., El-Deeb, A.A., Abdeldaim, A., Khan, Z.A.: On some generalizations of dynamic Opial-type inequalities on time scales. Adv. Differ. Equ. 2019, 323 (2019)

16. Saker, S.H.: Opial's type inequalities on time scales and some applications. Ann. Pol. Math. 104(3), 243-260 (2012)

17. Abdeljawad, T., Atici, F.M.: On the definitions of nabla fractional operators. Abstr. Appl. Anal. 2012, Article ID 406757 (2012)

18. Mohammed, P.O.: Hermite-Hadamard inequalities for Riemann-Liouville fractional integrals of a convex function with respect to a monotone function. Math. Meth. Appl. Sci., 1-11 (2019). https://doi.org/10.1002/mma.5784 
19. Mohammed, P.O., Sarikaya, M.Z., Baleanu, D.: On the generalized Hermite-Hadamard inequalities via the tempered fractional integrals. Symmetry 12, 595 (2020)

20. Fernandez, A., Mohammed, P.: Hermite-Hadamard inequalities in fractional calculus defined using Mittag-Leffler kernels. Math. Meth. Appl. Sci., 1-18 (2020). https://doi.org/10.1002/mma.6188

21. Mohammed, P.O., Abdeljawad, T.: Opial integral inequalities for generalized fractional operators with nonsingular kernel. J. Inequal. Appl. 2020, 148 (2020)

22. Tomovski, Z., Pečaric, J., Weighted, F.G.: Opial-type inequalities for fractional integral and differential operators involving generalized Mittag-Leffler functions. Eur. J. Pure Appl. Math. 10, 419-439 (2017)

23. Hilger, S.: Analysis on measure chains - a unified approach to continuous and discrete calculus. Results Math. 18, 18-56 (1990)

24. Bohner, M., Peterson, A.: Advances in Dynamic Equations on Time Scales. Birkhäuser, Boston (2003)

25. Agarwal, R.P., O'Regan, D., Saker, S.: Dynamic Inequalities on Time Scales. Springer, London (2014)

26. Gu, J., Meng, F.W.: Some new nonlinear Volterra-Fredholm type dynamic integral inequalities on time scales. Appl. Math. Comput. 245, 235-242 (2014)

27. Pachpatte, D.P.: Explicit estimates on integral inequalities with time scales. J. Inequal. Pure Appl. Math. 17, Article ID $143(2006)$

28. Rehák, P.: Hardy inequality on time scales and its application to half-linear dynamic equations. J. Inequal. Appl. 5, 495-507 (2005)

29. Mohammed, P.O.: Some integral inequalities of fractional quantum type. Malaya J. Mat. 4, 93-99 (2016)

30. Srivastava, H.M., Tseng, K.-L., Tseng, S.-J., LO, J.-C.: Some generalizations of Maroni's inequality on time scales. Math. Inequal. Appl. 14, 469-480 (2011)

31. Atici, F.M., Yaldız, H.: Convex functions on discrete time domains. Can. Math. Bull. 59, 225-233 (2016)

\section{Submit your manuscript to a SpringerOpen ${ }^{\circ}$ journal and benefit from:}

- Convenient online submission

- Rigorous peer review

- Open access: articles freely available online

- High visibility within the field

- Retaining the copyright to your article

Submit your next manuscript at $>$ springeropen.com 